J. Lake Sci. (湖泊科学), 2020, 32(6): 1817-1826

DOI 10. 18307/2020. 0621

(c) 2020 by Journal of Lake Sciences

\title{
鳑鲏对“蚌一草”关系的影响”
}

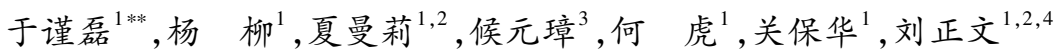

(1: 中国科学院南京地理与湖泊研究所湖泊与环境国家重点实验室,南京 210008)

(2: 暨南大学生态学系水生生物研究所,广州 510632)

(3: 同济大学环境科学与工程学院, 上海 200092)

(4:中国科学院大学中丹科研教育中心, 北京 100049)

摘 要: 重建沉水植物群落是修复浅水富营养化湖泊的关键. 河蚌可改善水下光照条件、促进沉水植物生长, 因此放养 河蚌常被用于沉水植物群落恢复的并行手段. 河蚌是鳑鲏产卵的重要基质, 因此河蚌可能促进鳑鲏种群发展, 而鳑鲏对 水生态系统的影响还尚不清楚. 本研究以密刺苦草、大䲠鱊和背角无齿蚌为研究对象, 通过中宇宙试验研究河蚌和鳑鲔 对附着藻的影响, 以及鳑鲏对水质和沉水植物生长的影响及机理. 结果发现: 鳑鲏显著增加了水体总磷、总溶解性磷、总 悬浮物和叶绿素 $a$ 浓度, 而对总氮和总溶解性氮浓度的影响不显著. 河蚌对苦草的相对生长率、总株数、根冠比及苦草最 大叶长均无显著影响, 而显著增加了苦草的单株平均生物量, 这可能与河蚌组较高的附着藻生物量有关. 鳑鲏未显著影 响河蚌与苦草间的关系, 但鳑鲏的出现显著增加了附着藻类生物量; 此外, 鳑鲏还降低了苦草的根冠比, 而增加了苦草 的最大叶长, 这可能与鳑鲏引起的营养盐和叶绿素 $a$ 浓度升高, 以及植物表面附着藻生物量显著升高有关. 鳑鲏属于小 型杂食性鱼类, 在长江中下游地区分布广泛, 与沉水植物关系密切, 且易在修复后的湖泊中形成优势鱼类, 因此在湖泊 修复和管理中应加强此类小型杂食性鱼类的监测与管理.

关键词: 大鯺魣; 河蚌; 密刺苦草; 湖泊生态修复;沉水植物;杂食性鱼类

\section{Effects of bitterling fish on the relationships between submerged macrophytes and mus- sel $^{*}$}

YU Jinlei ${ }^{1 * *}$, YANG Liu ${ }^{1}$, XIA Manli ${ }^{1,2}$, HOU Yuanzhang ${ }^{3}$, HE Hu ${ }^{1}$, GUAN Baohua ${ }^{1} \&$ LIU Zhengwen ${ }^{1,2,4}$

(1: State Key Laboratory of Lake Science and Environment, Nanjing Institute of Geography and Limnology, Chinese Academy of Sciences, Nanjing 210008, P.R.China)

(2: Department of Ecology and Institute of Hydrobiology, Jinan University, Guangzhou 510632, P.R.China)

(3: College of Environment Science and Engineering, Tongji University, Shanghai 200092, P.R.China)

(4: Sino-Danish Center for Education and Research, University of Chinese Academy of Sciences, Beijing 100049, P.R.China)

Abstract: Submerged macrophytes play an important role in stabilizing the water environments, thus restoration of submerged macrophytes has been the key to the restoration of shallow eutrophic lakes. Meanwhile, mussel stocking has been conducted as a parallel measure for submerged macrophytes restoration, because the filter-feeding mussels can improve and maintain a higher water clarity which it will enhance the growth of macrophytes. However, mussels, acting as an important spawning substrate for bitterling, may also facilitate the population development of bitterling fish (the common name for cyprinid fishes from the subfamily Acheilognathinae). The effects of bitterling fish on aquatic ecosystems are not clear. In the present study, we conducted an outdoor mesocosm experiment to study the effects of bitterling fish on water quality, the growth of submerged macrophytes and the attached periphyton, and the relationship between mussel and macrophytes. Three treatments were set with 4 replicates for each group, they are control (without mussel and bitterlings), mussels (with Sinanodonta woodiana), and mussel-bitterlings treatment (with both A. woodiana and Acheilognathus macropterus), meanwhile all the mesocosms were planted with equal density and biomass of Vallisne-

* 2019-10-31 收稿;2020-03-20 收修改稿.

国家水体污染控制与治理科技重大专项 (2017ZX07203-004) 和国家自然科学基金项目(41877415)联合资助.

** 通信作者; E-mail: jlyu@ niglas.ac.cn. 
ria denseserrulata. The results showed that the presence of bitterlings significantly increased the concentrations of total phosphorus, total dissolved phosphorus, suspended solids (TSS, OSS and ISS) and Chl. $a$ of phytoplankton, but not for total nitrogen and total dissolved nitrogen. At the end of the experiment, the growth rate (both $R G R$ and total individual), root-shoot biomass ratio, and maximum leaf length of macrophytes did not differ significantly between the mussel and control mesocosms, while the mean individual biomass of macrophytes in the mussel group was significantly higher than that of the controls which may be related to the higher biomass of periphyton on the surface of macrophytes leaves, though not significant compared with the controls. However, the presence of bitterlings did not significantly affect the mussel-macrophytes relationships. In comparison with the mussel's treatment, although bitterlings did not affect the $R G R$ and total individuals of macrophytes, the maximum length of leaves was higher in the mussel-bitterling mesocosms than mesocosms with mussels, while the root-shoot biomass ratio was lower. These changes may be caused by the much higher concentrations of nutrients, suspended solids, Chl. $a$ of phytoplankton and biomass of periphyton on the surface of leaves induced by the bitterlings which may boosted the growth of macrophytes leaves. Our results have important implications for lake management and restoration: bitterling is a small omnivorous fish which is widely distributed in lakes in the middle and lower reaches of Yangtze River. Moreover, they are highly correlated with submerged macrophytes and recovered quickly after lake restoration, so the monitoring and management of the small omnivorous fish, like bitterling in our study, should be strengthened when restoring and managing of shallow lakes.

Keywords: Bitterling; mussel; Vallisneria denseserrulata; lake restoration; submerged macrophytes; omnivorous fish

沉水植物在水生态系统中发挥重要作用 ${ }^{[1]}$, 它通过叶片吸收水体营养盐, 可有效降低水体营养盐浓度; 通过根系泌氧提高沉积物氧含量, 抑制磷的释放速率; 其发达的根系还可稳固沉积物, 抑制沉积物再悬 浮 ${ }^{[2-3]}$. 因此,在浅水湖泊生态修复中,沉水植物群落恢复被认为是湖泊修复的关键. 该技术也被广泛应用于 我国热带、亚热带湖泊生态修复实践,在一些外源污染得到一定控制的湖泊中取得较好效果 ${ }^{[4-8]}$, 其中惠州 西湖南湖的生态修复效果已稳定维持了 10 多年 ${ }^{[7]}$.

在沉水植物恢复初期,即使在沉水植物覆盖度较高的水体,营养盐也可维持在较高的浓度水平. 这主要 归因于长期处于富营养化状态的湖泊, 沉积物中的营养盐含量较高, 在适宜的环境条件下, 将不断的释放到 上覆水 ${ }^{[9-11]}$, 从而促进浮游植物生物量快速增长, 引起透明度下降, 影响沉水植物生长. 湖泊富营养化引起的 透明度下降, 被认为是沉水植物生物量下降和生物多样性损失的主要原因 ${ }^{[12]}$. 因此, 在沉水植物恢复初期, 为了维持适宜的光照条件, 加速和保障沉水植物群落恢复, 常将放养可通过滤食作用提高水体透明度 ${ }^{[13-16]}$ 、 促进沉水植物生长 ${ }^{[17]}$ 的河蚌作为其并行手段, 并已应用于湖泊生态修复 ${ }^{[4,8,18]}$. 而较好的水下光照, 还有利 于植物表面附着藻类的生长, 将会对沉水植物生物量和多样性产生显著的负面影响 ${ }^{[19-20]}$. 但河蚌对沉水植 物表面附着藻类的影响还未见相关报道.

河蚌是鳑鲔鱼类(鲤科鳑鲏亚科鱼类的统称)繁殖的重要基质,放养河蚌可能有利于鳑鲏种群的增加. 鳑鲏的受精卵必须在河蚌 (例如背角无齿蚌和三角帆蚌等 ${ }^{[21]}$ ) 的外套膜发育, 幼鱼在此生活一段时间后才 独自生活. 因此,放养的河蚌可通过携带鳑鲏受精卵和增加鳑鲏的产卵基质两种途径,补充或促进鳑鲏种群 的发展. 鳑鲔属于小型杂食性鱼类, 可摄食浮游动物、底栖动物、附着藻类、碎屑和沉水植物等 ${ }^{[5,22]}$. 而杂食 性鱼类可通过排泄、扰动沉积物和影响浮游动物群落结构和生物量等途径, 引起水体营养盐和浮游植物生 物量升高, 从而削弱水下光照 ${ }^{[23-26]}$, 影响沉水植物生长 ${ }^{[24]}$. 而鳑鲏对水质及沉水植物生长的影响还尚不 清楚.

杂食性鱼类在低纬度地区浅水湖泊中的物种丰度较高 ${ }^{[27]}$, 也是我国长江中下游湖泊的优势鱼类. 现有 研究多关注较大个体杂食性鱼类的影响 ${ }^{[23-26]}$, 对小型杂食性鱼类的关注较少. 而这些小型鱼类是某些生态 修复湖泊的优势种 ${ }^{[5,28]}$, 且放养肉食性鱼类也很难控制其数量 ${ }^{[4-5]}$. 因此, 了解此类小型杂食性鱼类对水生 态系统的影响, 将为浅水湖泊管理和生态修复提供理论参考. 本研究通过设置中宇宙试验系统, 以密刺苦草 (Vallisneria denseserrulata)、大鯺鱊 (Acheilognathus macropterus) 和背角无齿蚌 (Sinanodonta woodiana) 为研究 对象,探讨大鳍鱊(鳑鲏) 对水质、密刺苦草生长和“蚌一草”关系的影响. 


\section{1 材料与方法}

\section{1 试验设置}

本试验在位于苏州东山镇的中国科学院南京地理与湖泊研究所太湖湖泊生态系统实验站的池塘中进 行 (东山站). 试验采用的试验系统为高密度聚乙烯塑料桶 (容积: $130 \mathrm{~L}$; 高: $66 \mathrm{~cm}$; 上口直径: $56 \mathrm{~cm}$; 底 部直径: $45 \mathrm{~cm}$ ). 每个桶内加人 $10 \mathrm{~cm}$ 混匀的沉积物 (采自东山站池塘, 用孔径为 $0.5 \mathrm{~cm}$ 的篮网过滤) 和 100 $\mathrm{L}$ 经 $64 \mu \mathrm{m}$ 浮游生物网过滤的湖水; 同时, 也将提前准备好的蓄水桶 $(1000 \mathrm{~L})$ 装满, 用于补充各试验系统在 试验期间流失的水分 (自然蒸发和采样用水).

在试验开始之前, 向 12 个中宇宙系统中分别栽植 10 株密刺苦草 ( 以下简称苦草), 其平均叶长为 $20.5 \pm$ $1.7 \mathrm{~cm}$ 、平均生物量为 $81.8 \pm 8.1 \mathrm{~g} / \mathrm{m}^{2}$. 本试验共设置 3 个处理组, 分别为对照组 (仅栽植苦草) 、河蚌组 (苦 草+放养河蚌) 和河蚌+鳑鲏组 (苦草+河蚌+鳑鲏), 每个处理组设置 4 个重复. 苦草种植 7 天后, 对照组的试 验系统不再添加任何试验材料; 向河蚌组的试验系统中加人 1 只背角无齿蚌 (以下简称河蚌), 其生物量为 $264.4 \pm 2.5 \mathrm{~g} / \mathrm{m}^{2}$; 而在放养鳑鲏的试验系统中, 不仅添加相同数量和相近生物量 $\left(266.7 \pm 5.0 \mathrm{~g} / \mathrm{m}^{2}\right)$ 的河蚌, 而且还加人 3 尾规格相近的大鳍鱊: 平均全长 $7.5 \pm 0.5 \mathrm{~cm}$; 平均湿重 $9.1 \pm 0.2 \mathrm{~g}$; 生物量 $39.9 \pm 2.7 \mathrm{~g} / \mathrm{m}^{2}$, 经 分析: 系统间的大鳍魣规格与生物量不存在显著性差异. 本试验采用的鱼类密度 $\left(8.6 \mathrm{ind} . / \mathrm{m}^{2}\right)$ 接近自然湖 泊中的小型鱼类密度 $\left(8.7 \mathrm{ind} . / \mathrm{m}^{2}\right)^{[29]}$; 而背角无齿蚌的生物量低于 $2006-2007$ 年太湖蚌类的总平均生物 量 $\left(647.4 \mathrm{~g} / \mathrm{m}^{2}\right)^{[30]}$. 鳑鲏和河蚌均在试验开始时同时投放到相应的试验系统. 本试验周期为 42 天, 在 4-5 月 (4月 8 日至 5 月 20 日) 开展试验. 试验期间的平均水温为 $19.4 \pm 2.2^{\circ} \mathrm{C}$.

\section{2 样品采集与分析}

在试验开始的第 $0 、 7 、 14 、 21 、 28 、 35$ 和 42 天测定水体的营养盐、悬浮质和叶绿素 $a($ Chl. $a)$ 浓度; 试验结 束时, 测定植物的各项生长指标和植物表面附着藻生物量. 每次水样采集时, 用 $2.5 \mathrm{~L}$ 的有机玻璃采水器, 收 集 $5 \mathrm{~L}$ 不同水层的上覆水,然后准备 $2 \mathrm{~L}$ 混合均匀的水样回试验室分析水体不同形态的氮、磷营养盐浓度、 Chl. $a$ 浓度和不同形态的悬浮物浓度, 最后将剩余水样倒回试验系统. 每次采样结束或当系统水位变化明显 时,我们用提前准备好的湖水将水位恢复至试验初始水平.

总氮 (TN) 、溶解性总氮 (TDN)、总磷 (TP)、溶解性总磷 (TDP) 浓度的分析方法参照《湖泊富营养化调查 规范 $\rangle^{[31]}$. 用醋酸纤维膜过滤 $0.5 \sim 1 \mathrm{~L}$ 水样, 将滤膜转人离心管, 加人 $10 \mathrm{~mL} 90 \%$ 的丙酮溶液, 充分震荡后置 于 $4^{\circ} \mathrm{C}$ 暗处反应 $18 \sim 24 \mathrm{~h}$; 随后, 经 $3500 \mathrm{r} / \mathrm{min}$ 离心后, 取上清液, 用紫外分光光度计测定其在 750、663、645 和 $630 \mathrm{~nm}$ 波长下的吸光值,计算水样中的 Chl. $a$ 浓度.

用已知重量 $\left(W_{0}\right)$ 的 Whatman $\mathrm{GF} / \mathrm{C}$ 玻璃纤维膜抽滤 $500 \sim 1000 \mathrm{~mL}$ 水样, 随后将膜置于 $60^{\circ} \mathrm{C}$ 烘箱内, 48 $\mathrm{h}$ 后通过万分之一的天平获得膜重 $\left(W_{1}\right), W_{1}$ 与 $W_{0}$ 的差值即为水体总悬浮质 ( TSS) 浓度. 然后, 将膜置于 $450^{\circ} \mathrm{C}$ 的马弗炉中灼烧 $4 \mathrm{~h}$, 取出称重 $\left(W_{3}\right)$, 通过 $W_{1}$ 和 $W_{3}$ 的重量差获得总悬浮有机质 (OSS) 浓度; 而 $W_{1}$ 与 $W_{0}$ 的差值即为总悬浮无机质 (ISS) 的浓度.

试验结束时, 将每个试验桶中所有苦草取出, 测量每个桶的总株数、总生物量和最大叶长. 苦草根冠比 测定时, 从每个试验系统中随机选取 10 株植物, 在实验室将表面附着物洗净后, 置于 $60^{\circ} \mathrm{C}$ 烘箱中烘 $48 \mathrm{~h}$, 然 后分别称量每株植物的根和叶片重量, 获得植物的根冠比值. 苦草叶片表面附着藻样品收集时, 从每个试验 系统中随机选取 3 株植物, 然后从每株植物上随机摘取 1 片叶片. 为了保证所收集的附着藻来自同一水层, 避免不同水层附着藻生物量的差异, 我们选取苦草叶片离地 (沉积物) $20 \sim 30 \mathrm{~cm}$ 处的叶片测定其附着藻生 物量 (用 Chl. $a$ 浓度表示). 然后, 将获得的苦草叶片用牙刷将其表面的附着藻刷人烧杯中. 最后, 用量筒测 量附着藻液体积后, 用 $\mathrm{GF} / \mathrm{C}$ 膜抽滤一定体积混匀的附着藻液 (体积视藻液浓度而定), 参照水体 Chl. $a$ 浓度 的测定方法获得附着藻类生物量 $\left(\mu \mathrm{g} / \mathrm{cm}^{2}\right)$.

苦草的单株平均生物量 $(\mathrm{g} /$ 株) 通过每个系统的苦草总重量和总株数获得. 苦草相对生长率 $(R G R$, $(\mathrm{mg} /(\mathrm{g} \cdot \mathrm{d}))$ 的计算公式为:

$$
R G R=1000 \times \ln \left(W_{\mathrm{f}} / W_{\mathrm{i}}\right) / d
$$

式中, $W_{\mathrm{f}}$ 和 $W_{\mathrm{i}}$ 分别是苦草结束和初始总生物量 $(\mathrm{g}) ; d$ 是试验持续的天数. 


\section{3 数据分析}

本研究的所有数据统计分析均在 SPSS 22.0 软件中进行. 通过单因素重复测量方差分析 (rmANOVA), 分别比较对照组与河蚌组和河蚌组与河蚌+鳑鲏组间的 TN、TDN、TP、TDP、Chl. $a$ 、TSS、OSS 和 ISS 浓度差异, 其中组内比较参照一元方差分析中 Greenhouse-Giesser 校正后的结果. 试验结束时, 对照组与河蚌组和河蚌 组与河蚌+鳑鲔组间苦草的 $R G R$ 、总株数、根冠比和平均单株生物量, 以及植物表面附着藻类生物量采用 $t-$ test 方法进行统计分析.

\section{2 结果}

\section{1 水体营养盐浓度}

对照组 $(P<0.01)$ 、河蚌组 $(P<0.05)$ 和河蚌+鳑鲏组 $(P<0.05)$ 的水体 TN 浓度随时间变化显著, 但对照 组与河蚌组和河蚌组与河蚌十鳑鲔组间的 TN 浓度差异不显著 $(P>0.05)$ (图 1a); TDN 浓度在对照组 $(P<$ $0.01)$ 、河蚌组 $(P<0.05)$ 和河蚌+鳑鲔组 $(P<0.01)$ 中随时间的变化显著, 但各处理组间 TDN 的浓度也无显著 性差异 $(P>0.05)$ (图 1b).

对照组和河蚌组的 TP 浓度变化趋势相似, 随时间的变化趋势显著 $(P<0.05)$; 但河蚌组与对照组的 TP 浓度在整个试验过程中均无显著差异 $(P>0.05)$ (图 1c). 鳑鲏组的 TP 浓度在整个过程中呈逐渐升高的趋势 $(P<0.01)$; 其中, 第 $14 、 21$ 和 28 天的浓度均显著高于河蚌组 $(P<0.01)$, 而其他采样时间的 TP 浓度与河蚌 组间均无显著差异 $(P>0.05)$ ( 图 1c).
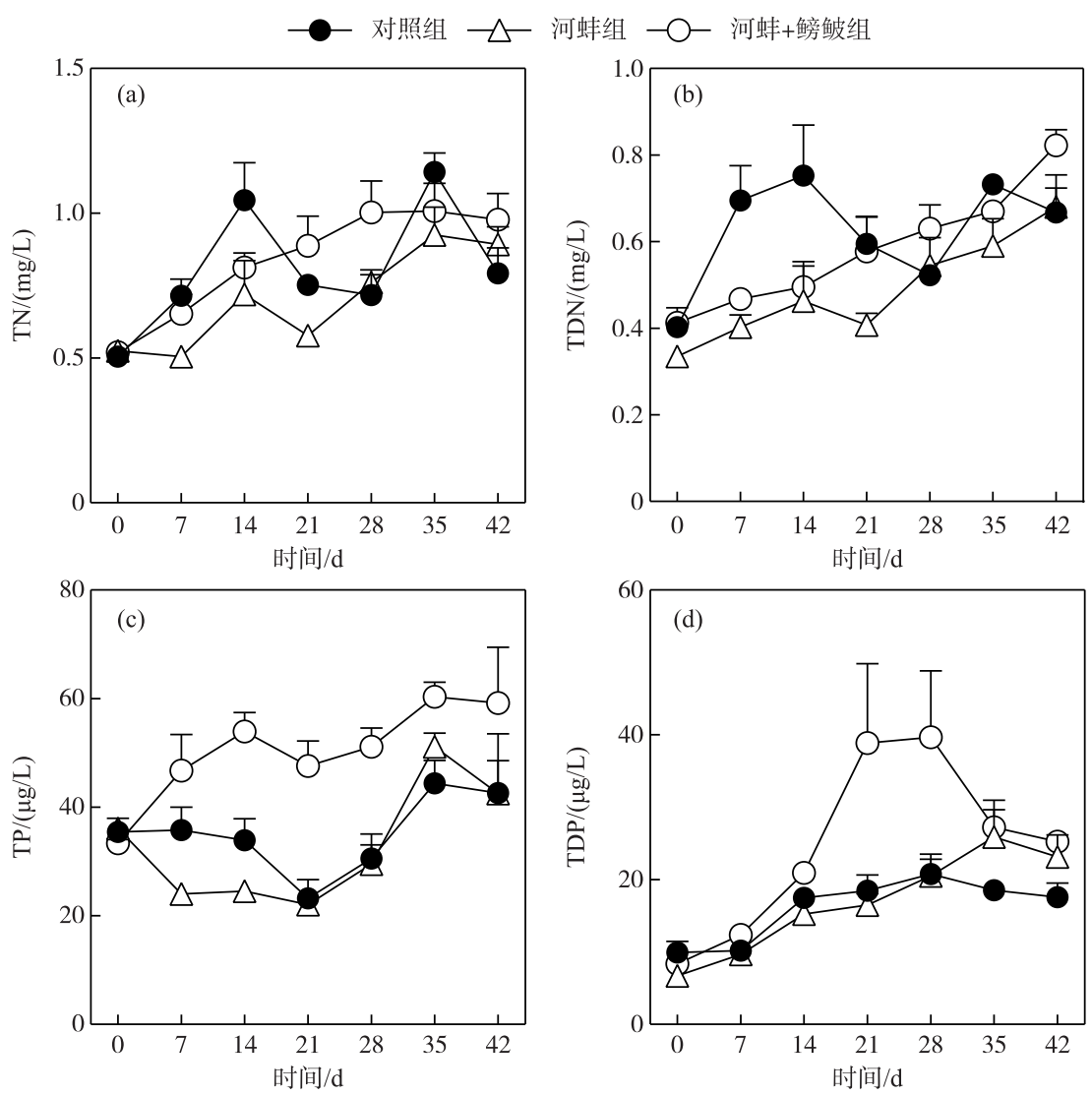

图 1 试验期间不同处理组的总氮、总溶解氮、总磷和总溶解磷浓度随时间的变化

Fig. 1 Concentrations of total nitrogen, total dissolved nitrogen, total phosphorus and total dissolved phosphorus in different treatments during the experiment 
在整个试验周期内,对照组的 TDP 浓度变化不显著 $(P>0.05)$, 而河蚌组和河蚌+鳑鲏组的 TDP 浓度随时间 变化显著 $(P<0.05)$ (图 1d). 河蚌+鳑鲏组的 TDP 浓度仅 在试验开始的第 21 天时显著高于河蚌组 $(P<0.01)$, 而在 其他采样时间中, 河蚌组与对照组以及河蚌十鳑鲏组与河 蚌组间的 TDP 浓度均无显著性差异 $(P>0.05)$ (图 1d).

\section{2 水体 Chl. $a$ 浓度}

在本试验中, 3 个处理组的 Chl. $a$ 浓度随时间的变化 显著 $(P<0.05)$ (图 2). 对照组和河蚌组间的 Chl. $a$ 浓度, 在整个试验过程中均无显著性差异 $(P>0.05)$. 而河蚌十鳑 鲏组的 Chl. $a$ 浓度显著高于河蚌组 $(P<0.001)$; 在试验的 第 7 天, 河蚌十鳑鲏组的 Chl. $a$ 浓度显著高于河蚌组 $(P<$ $0.0001)$, 而在其他采样时间这两组的 Chl. $a$ 浓度差异不 显著 $(P>0.05)$ ( 图 2).

\section{3 水体悬浮质浓度}

对照组、河蚌组和河蚌+鳑鲏组的 TSS 浓度随时间变 化显著 $(P<0.05)$. 对照组的 TSS 浓度在试验开始后逐渐下

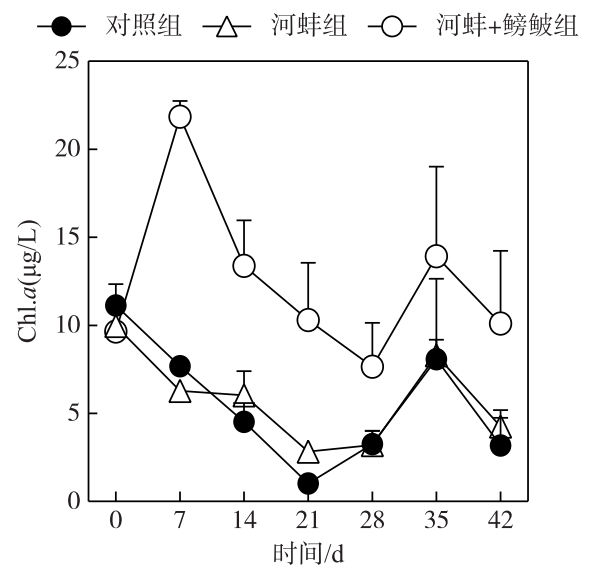

图 2 试验期间不同处理组的 水体叶绿素 $a$ 浓度

Fig.2 Concentrations of chlorophyll- $a$ in different treatments during the experiment

降,但仅第 21 和 35 天的 TSS 浓度显著低于初始浓度 $(P<0.05)$. 河蚌组的 TSS 浓度呈先升后降的变化趋势, 其 中河蚌组第 7 天的 TSS 浓度显著高于初始值 $(P<0.05)$, 而第 21 和 28 天的 TSS 浓度则显著低于初始浓度 $(P<$ 0.05 ) (图 3a). 河蚌+鳑鲏组的 TSS 浓度也呈先升后降的趋势, 其第 7 天的浓度, 显著高于初始浓度 $(P<0.05)$, 而其他采样时间的 TSS 浓度均与初始浓度无显著差异 $(P>0.05)$.

试验开始时, 河蚌组与对照组和河蚌+鳑鲏组与河蚌组间的 TSS 浓度均相近 $(P>0.05)$. 河蚌+鳑鲏组的 TSS 浓度从第 7 天开始直至第 28 天, 一直显著高于河蚌组 $(P<0.05)$. 河蚌组与对照组间的 TSS 浓度仅在试 验开始的前两周内存在显著性差异 $(P<0.01)$, 而在其他采样时间点的差异不显著 $(P>0.05)$ （图 3a).

此外, 对照组和河蚌组的 OSS 浓度随时间的变化显著 $(P<0.05)$, 而河蚌+鳑鲔组的 OSS 浓度随时间的 变化不显著 $(P>0.05)$. 试验期间, 河蚌+鳑鲏组的 OSS 浓度显著高于河蚌组 $(P<0.0001)$ (图 $3 \mathrm{~b})$. 对照组的 ISS 浓度随时间变化不显著 $(P>0.05)$, 而河蚌组 $(P<0.05)$ 和河蚌+鳑鲏组 $(P<0.05)$ 的 ISS 浓度随时间变化 显著 (图 3c). 河蚌+鳑鲏组的 ISS 浓度显著高于河蚌组 $(P<0.0001)$, 而河蚌组与对照组间的 ISS 浓度在整个 试验期间均无显著性差异 $(P>0.05)$ ( 图 3c).
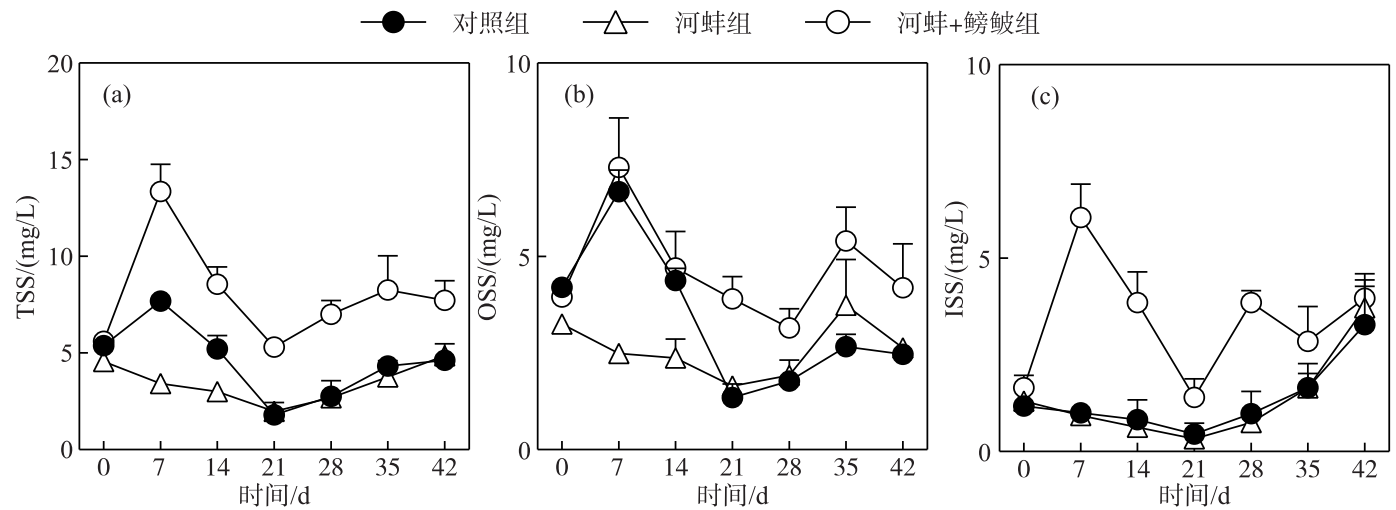

图 3 试验期间不同处理组的总悬浮质、总有机悬浮质和总无机悬浮质浓度随时间的变化

Fig. 3 Concentrations of total suspended solids, organic suspended solids and inorganic suspended solids in different treatments during the experiment 


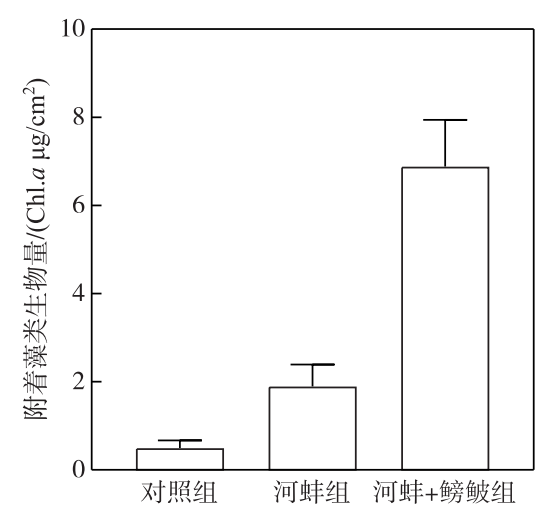

图 4 密刺苦草叶片表面附着藻类生物量

Fig.4 The biomass of periphyton on the surface of $V$. denseserrulata

\section{4 附着藻类}

试验结束时, 河蚌组密刺苦草叶片表面附着藻类生物量高 于对照组, 但两者间的差异不显著 $(P>0.05)$; 而河蚌+鳑鲏组 的附着藻类生物量显著高于河蚌组 $(P<0.01)$ ( 图 4).

\section{5 密刺苦草}

试验结束时, 对照组中苦草的 $R G R$ 略高于河蚌组 $(P>$ $0.05)$; 同时, 河蚌+鳑鲏组与河蚌组间苦草的 $R G R$ 差异也不显 著 $(P>0.05$, 图 5a). 河蚌+鳑鲏组的苦草总株数 ( $95 \pm 8$ 株) 高于 河蚌组 ( $82 \pm 10$ 株), 但差异不显著 $(P>0.05)$; 对照组中苦草的 平均总株数为 $86 \pm 13$ 株, 与河蚌组间的差异不显著 $(P>0.05$, 图 $5 b)$.

河蚌组的苦草根冠比低于对照组 $(P>0.05)$; 而鳑鲏的出 现, 对苦草的根冠比影响不显著, 其(河蚌+鳑鲏组) 根冠比略低 于河蚌组 $(P>0.05$ ) (图 6a). 河蚌+鳑鲏组的苦草最大叶长高于 对照组 $(P>0.05)$; 河蚌组的最大叶长高于对照组, 且差异显著 $(P>0.05)$ (图 6b). 此外, 对照组的苦草平均单株生物量显著低于河蚌组 $(P<0.01)$, 但河蛙组与河蛙 + 鳑鲏 组间不存在显著性差异 $(P>0.05)$ ( 图 6c).
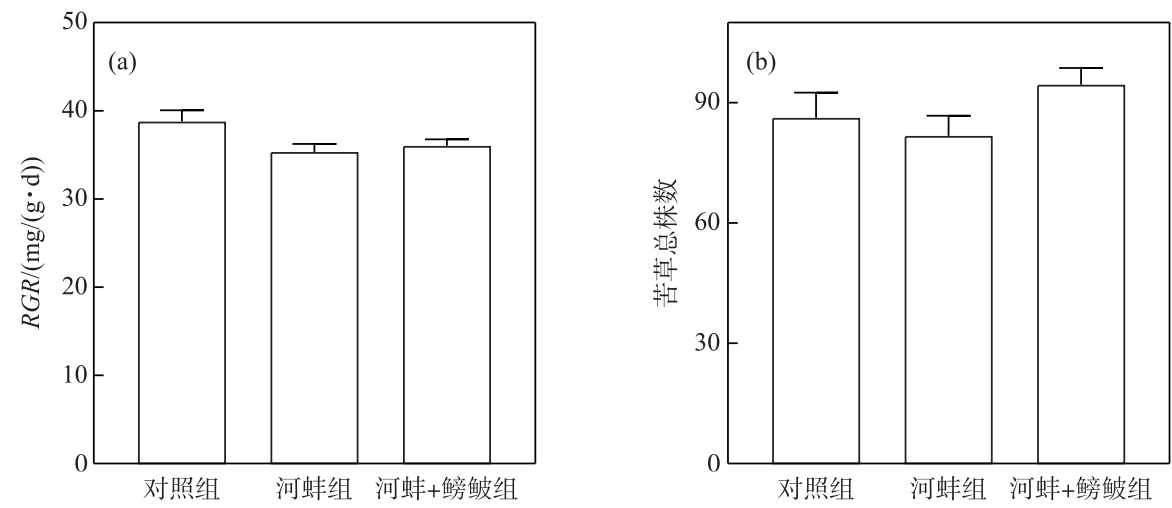

图 5 密刺苦草在试验结束时的相对生长率 $(R G R)$ 和总株数

Fig.5 The relative growth rate (RGR) and total plant individuals of $V$. denseserrulata at the end of the experiment
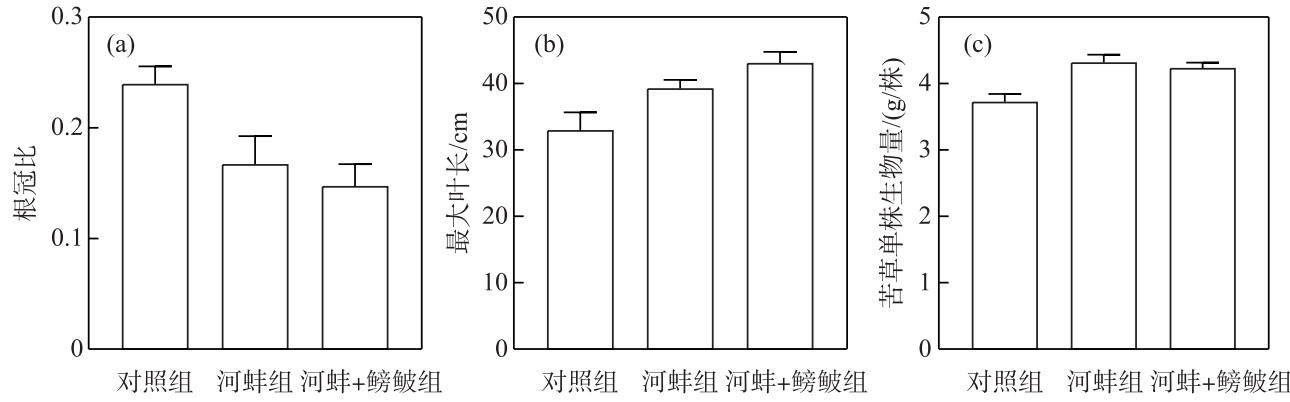

图 6 试验结束时密刺苦草的根冠比、最大叶长和单株平均生物量

Fig.6 Root-shoot dry biomass ratio, maximum leaf length and mean individual biomass of $V$. denseserrulata at the end of the experiment 


\section{3 讨论}

目前关于鳑鲏对水质或生态系统影响的研究相对较少, 仅喻倩等 ${ }^{[32]}$ 通过室内试验报道了高密度鳑鲏(中 华鳑鲏)对水质等的影响, 发现较高密度鳑鲏可显著增加水体 TN、TP 和氨氮浓度. 而在我们的中宇宙原位生 态系统中,鳑鲏对 TP 和 TDP 浓度的影响显著, 而对 TN 和 TDN 浓度的影响不显著. 这可能与试验系统中存在 沉水植物有关: 一方面, 由于系统初始的氮浓度相对较低, 密刺苦草生长过程中可直接吸收水体营养盐, 降低 水体营养盐浓度; 另一方面, 沉水植物表面的附着物可为硝化和反硝化细菌提供生境, 提高水体的脱氮能 力 $^{[33]}$. 此外, 沉水植物还可通过影响水体 $\mathrm{pH}$ 和溶解氧的浓度, 促进氨的挥发 ${ }^{[34]}$. 因此, 沉水植物通过直接吸收 利用和间接影响氮素的地球化学循环速率等方式, 保持系统中较低的氮浓度. 而河蚌+鳑鲏组中较高的磷浓 度, 可能主要与鳑鲏通过扰动沉积物间接促进沉积物磷释放和直接排泄引起磷浓度有关. 排泄和扰动沉积物 是杂食性鱼类影响水体营养盐浓度的主要途径, 这些行为在底栖杂食性鱼类中较为常见 ${ }^{[23-24,35]}$.

杂食性鱼类通过扰动沉积物, 可引起水体悬浮物浓度升高 ${ }^{[26,36-37]}$. 在本研究中, 河蚌组的悬浮物浓度一 直处于较低水平; 而大鳍鱊的出现则显著增加了 TSS 浓度, 其中 OSS 和 ISS 的浓度也显著高于河蚌组. 河 蚌+鳑鲏组中 OSS 浓度的升高, 主要由于该组的营养盐(主要是 TP 和 TDP) 浓度较高, 促进了浮游植物生 长, 河蚌+鳑鲔组的浮游植物生物量 (Chl. a ) 显著高于其他处理组也佐证了这一点. 此外, 浮游动物是鳑鲏的 重要食物来源 ${ }^{[5,22,38]}$, 鳑鲏可能通过降低枝角类等浮游动物的生物量, 削弱浮游动物对浮游植物的下行控 制, 是引起河蚌+鳑鲏组 Chl. $a$ 浓度显著高于其他两组的原因之一, 这也是其他杂食性鱼类影响水体 Chl. $a$ 浓

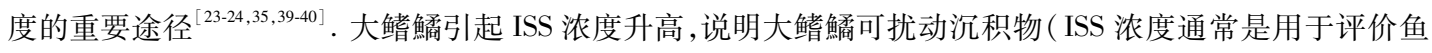
类扰动沉积物强弱的指标), 这些影响在试验初期较为明显, 而在试验中后期随着密刺苦草生物量不断增 加, 抑制了鳑鲏对沉积物的扰动作用; 此外, 在背角无齿蚌滤食的共同作用下, ISS 浓度逐渐下降,并在试验 结束时与河蚌组间无显著差异 (图 3c). 而在大鲲鱊与河蚌共存的试验系统中, 河蚌未能有效地抑制鳑鲏引 起的水体 Chl. $a$ 和 OSS 浓度升高. 这可能主要与本研究中较低的河蚌与鳑鲏生物量比 (6.7:1) 有关; 当河蚌 与鳑鲏的生物量比高于 10:1 时, 河蚌有效抑制了水体 Chl. $a$ 和 OSS 浓度的升高 (于谨否, 未发表数据).

沉水植物生长与水下光照密切相关, 而较高的悬浮质浓度和附着藻类生物量可阻碍沉水植物对光的获 取, 从而影响沉水植物生长 ${ }^{[19,41]}$. 有研究发现, 沉水植物表面附着藻类生物量随着水体营养盐浓度的升高而 逐渐增加 ${ }^{[39-40]}$. 在自然湖泊中, 研究者发现沉水植物表面附着藻类生物量越高的湖泊, 其沉水植物生物量、 覆盖度和多样性越低 ${ }^{[44]}$. 而在削减植物表面附着藻类生物量后, 植物的光合作用效率明显增加 ${ }^{[45]}$. 在我们 的研究中, 河蚌组与对照组间的营养盐、悬浮质和 Chl. $a$ 浓度均无明显差异, 即使河蚌组的附着藻类生物量 略高于对照组, 但并没有抑制河蚌组密刺苦草的生长与种群扩张, 其 $R G R$ 和植物总株数与对照组间无显著 性差异 (图 5). 而河蚌+鳑鲏组的附着藻生物量显著高于其他处理组. 这主要由于河蚌+鳑鲏组的营养盐浓 度较高, 促进了附着藻的生长, 因为附着藻生物量可随营养盐的升高而显著增加 ${ }^{[42,46]}$. 但在试验结束时, 鳑 鲏的出现未对密刺苦草的 $R G R$ 和总株数产生显著影响, 说明本试验采用的鳑鲏密度在短期内 ( 42 天) 未对 苦草的生长产生显著的负面影响. 而鳑鲏对沉水植物生长的长期影响还需进一步研究.

河蚌显著增加了苦草的单株生物量, 说明在植物表面附着较高的藻类生物量时, 密刺苦草的生长策略 发生了变化. 我们发现密刺苦草主要通过增加叶长和地上部分生物量的方式来应对鳑鲏试验系统内较弱的 水下光照条件 (较高的 Chl. $a$ 和 TSS 浓度), 因为河蚌+鳑鲏组的苦草叶片较长而根冠比较低 (较高的地上部 分生物量). 这可以解释为什么鳑鲏的出现未显著影响密刺苦草的相对生长率. 此外, 试验期间系统内的营 养盐浓度远远低于文明章等 ${ }^{[47]}$ 报道的限制苦草生物量和生长率的浓度水平 ( TN : $10 \mathrm{mg} / \mathrm{L}$ 和 TP: $0.4 \mathrm{mg} /$ $\mathrm{L})$, 这可能也是本试验各处理组间苦草 $R G R$ 差异不显著的另一原因.

本研究结果对浅水湖泊生态修复与生态系统管理具有重要的指导意义. 鳑鲏幼鱼主要以浮游动物为 食, 而成鱼则主要以沉水植物为食 ${ }^{[5]}$. 在我国的一些亚热带浅水湖泊生态修复中, 肉食性鱼类 (如䰻和乌鳢) 很难控制类似鳑鲏的小型杂食性鱼类的数量 ${ }^{[4-5]}$, 因为这些肉食性鱼类对小型杂食性鱼类的摄食比例较 低 ${ }^{[5]}$. 因此, 这些小型杂食性鱼类容易发展成为生态修复湖泊的优势鱼类. 在我们的短期受控试验中 (42 天), 虽然鳑鲏的出现 (河蚌+鳑鲏组组) 未显著影响密刺苦草的生长和繁殖, 但鳑鲏不仅引起水体营养盐和 
Chl. $a$ 浓度升高, 而且还促进了植物表面附着藻的生长. 而过多的附着藻将通过遮光效应影响沉水植物生 长, 因此从湖泊生态修复效果的长效维持角度考虑, 过多的鳑鲏可能会进一步加剧植物表面附着藻的生长, 阻碍沉水植物生长和种群发展; 此外, 虽然鳑鲏可直接摄食沉水植物 ${ }^{[5]}$, 但由于受口裂大小的限制, 可能对 不同植物种类或同一种类的不同部位 (嫩芽、嫩叶、种子等) 表现出较强的选择性, 将会直接影响沉水植物生 长和种间竞争模式. 因此, 在湖泊生态修复中, 应加强鳑鲏等小型杂食性鱼类的监测与管理; 并开展进一步 研究,评估鳑鲏等小型杂食性鱼类在浅水湖泊生态系统中的地位与作用.

\section{4 结论}

1) 在密刺苦草为主的系统中, 与对照组相比, 背角无齿蚌 (河蚌组) 的出现未显著改变水体营养盐、Chl. $a$ 和悬浮颗粒物浓度.

2 ) 河蚌对密刺苦草的生长 (RGR)、繁殖 (总株数)、根冠比和最大叶长均无显著性影响, 而河蚌显著增加 了苦草的单株生物量 $(P<0.01)$; 此外, 苦草叶片表面附着藻生物量也高于对照组 (但差异不显著, $P>0.05)$.

3 ) 鳑鲏 (大鯺鱊) 的出现 (河蚌+鳑鲏组组), 不仅引起试验系统营养盐、Chl. $a$ 和悬浮物浓度显著升高, 而且还促进了植物表面附着藻的生长, 其附着藻生物量显著高于河蚌组.

4) 虽然河蚌+鳑鲏组的水体营养盐和植物表面附着藻生物量均显著高于河蚌组,但这并未显著影响密 刺苦草的生长和繁殖. 然而从湖泊生态修复效果的长效维持角度考虑, 过多的鳑鲏可能会进一步加剧水体 营养盐和 Chl. $a$ 浓度升高, 促进植物表面附着藻的生长, 通过遮光效应 (较高的浮游植物和附着藻类生物量) 阻碍沉水植物生长和种群发展. 因此, 在湖泊生态修复中, 应加强鳑鲏等小型杂食性鱼类的监测与管理; 并 开展进一步研究,评估鳑鲏等小型杂食性鱼类在浅水湖泊生态系统中的地位与作用.

致谢：感谢甄伟和王明飞对受控试验和实验室工作的支持.

\section{5 参考文献}

[ 1 ] Carpenter SR, Lodge DM. Effects of submersed macrophytes on ecosystem processes. Aquatic Botany, 1986, 26: 341-370. DOI: $10.1016 / 0304-3770$ ( 86) 90031-8.

[ 2 ] Scheffer M, Hosper SH, Meijer ML et al. Alternative equilibria in shallow lakes. Trends in Ecology \& Evolution, 1993,8 (8) : 275-279. DOI: 10.1016/0169-5347 (93) 90254-M.

[ 3 ] Horppila J, Nurminen L. Effects of submerged macrophytes on sediment resuspension and internal phosphorus loading in Lake Hiidenvesi ( southern Finland). Water Research, 2003, 37 (18) : 4468-4474. DOI: 10.1016/S0043-1354 ( 03 ) 00405-6.

[ 4 ] Chen KN, Bao CH, Zhou WP. Ecological restoration in eutrophic Lake Wuli: A large enclosure experiment. Ecological Engineering, 2009, 35(11) : 1646-1655. DOI: 10.1016/j.ecoleng.2008.10.009.

[ 5 ] Yu JL, Liu ZW, He H et al. Submerged macrophytes facilitate dominance of omnivorous fish in a subtropical shallow lake: implications for lake restoration. Hydrobiologia, 2016, 775: 97-107. DOI: 10.1007/s10750-016-2717-7.

[6 ] Yu JL, Liu ZW, Li KY et al. Restoration of shallow lakes in subtropical and tropical China: response of nutrients and water clarity to biomanipulation by fish removal and submerged plant transplantation. Water, 2016, 8: 438. DOI: 10. $3390 /$ w8100438.

[ 7 ] Liu ZW, Hu JR, Zhong P et al. Successful restoration of a tropical shallow eutrophic lake: Strong bottom-up but weak topdown effects recorded. Water Research, 2018, 146(1) : 88-97. DOI: 10.1016/j.watres.2018.09.007.

[ 8 ] Gao HL, Qian X, Wu HF et al. Combined effects of submerged macrophytes and aquatic animals on the restoration of a eutrophic water body-A case study of Gonghu Bay, Lake Taihu. Ecological Engineering, 2017, 102: 15-23. DOI: 10. 1016/j. ecoleng.2017.01.013.

[ 9 ] Søndergaard M, Liboriussen L, Pedersen AR et al. Lake restoration by fish removal: short- and long-term effects in 36 Danish lakes. Ecosystems, 2018, 11: 1291-1305. DOI: 10.2307/40296369.

[10] Søndergaard M, Bjerring R, Jeppesen E. Persistent internal phosphorus loading during summer in shallow eutrophic lakes. Hydrobiologia, 2013, 710: 95-107. DOI: 10.1007/s10750-012-1091-3.

[11] Nürnberg GK, Tarvainen M, Ventelä AM et al. Internal phosphorus load estimation during biomanipulation in a large polymictic and mesotrophic lake. Inland Waters, 2012, 2 : 147-162. DOI : 10.5268/IW-2.3.469. 
[12] Lauridsen TL, Jensen JP, Jeppesen E et al. Response of submerged macrophytes in Danish lakes to nutrient loading reductions and biomanipulation. Hydrobiologia, 2003, 506-509: 641-649. DOI: 10.1023/b:hydr.0000008633.17385.70.

[13] Wu ZK, Qiu XC, Zhang XF et al. Effects of Anodonta woodiana on water quality improvement in restoration of eutrophic shallow lakes. J Lake Sci, 2018, 30(6) : 1610-1615. DOI: 10.18307/2018.0612. [ 吴中奎, 邱小常, 张修峰等. 富营养 化浅水湖泊生态修复中背角无齿蛙 (Anodonta woodiana) 对水质改善的影响. 湖泊科学, 2018, 30 (6) : 1610-1615.]

[14] Johengen TH, Nalepa TF, Fahnenstiel GL et al. Nutrient changes in Saginaw Bay, Lake Huron, after the establishment of the zebra mussel (Dreissena polymorpha). Journal of Great Lakes Research, 1995, 21 (4) : 449-464. DOI: 10.1016/ s0380-1330 ( 95$)$ 71058-9.

[15] Wu QL, Chen YW, Liu ZW. Filtering capacity of Anodanta woodiana and its feeding selectivity on phytoplankton. Chinese Journal of Applied Ecology, 2005, 16(12) : 2423-2427. [吴庆龙, 陈宇炜, 刘正文. 背角无齿蚌对浮游藻类的滤食选 择性与滤水率研究. 应用生态学报, 2005, 16(12) : 2423-2427.]

[16] Yang DM, Chen YW, Liu ZW et al. Top-down effects of Anodonta woodiana on nutrient concentration \& phytoplankton community composition in a microcosm ecosystem. J Lake Sci, 2008, 20(2) : 228-234. DOI: 10.18307/2008.0215. [ 杨东 妹, 陈宇炜, 刘正文等. 背角无齿蚌滤食对营养盐和浮游藻类结构影响的模拟. 湖泊科学, 2008, 20 (2): 228-234.]

[17] He H, Liu X, Liu XL et al. Effects of cyanobacterial blooms on submerged macrophytes alleviated by the native Chinese bivalve Hyriopsis cumingii: A mesocosm experiment study. Ecological Engineering, 2014, 71: 363-367. DOI: 10.1016/j. ecoleng.2014.07.015.

[18] Qin B. A large-scale biological control experiment to improve water quality in eutrophic Lake Taihu, China. Lake and Reservoir Management, 2013, 29(1) : 33-46.

[19] Jones JI, Young JO, Eaton JW et al. The influence of nutrient loading, dissolved inorganic carbon and higher trophic levels on the interaction between submerged plants and periphyton. Journal of Ecology, 2002, 90: 12-24. DOI: 10. 2307/3072315.

[20] Sand-Jensen K, Borum J. Interactions among phytoplankton, periphyton, and macrophytes in temperate freshwaters and estuaries. Aquatic Botany, 1991, 41(1-3) : 137-175. DOI: 10.1016/0304-3770(91) 90042-4.

[21] Shen JZ. Experimental investigations into some reproduction aspects of Chinese bitterling Rhodeus sinensis. Journal of Huazhong Agricultural University, 2000, 19(5) : 494-496. DOI: 10.1016/j.cattod.2015.09.021. [沈建忠. 中华鳑鲏 Rhodeus sinensis 繁殖习性的初步观察. 华中农业大学学报, 2000, 19(5): 494-496.]

[22] Koutrakis ET, Kokkinakis AK, Tsikliras AC et al. Characteristics of the European bitterling Rhodeus amarus (Cyprinidae) in the Rihios river, Greece. Journal of Freshwater Ecology, 2003, 18 (4): 615-624. DOI: 10. 1080/02705060. 2003.9664003.

[23] He H, He E, Yu JL et al. Does turbidity induced by Carassius carassius limit phytoplankton growth? A mesocosm study. Environmental Science Pollution Research, 2017, 24: 5012-5018. DOI: 10.1007/s11356-016-8247-z.

[24] Gu J, Jin H, He H et al. Effects of small-sized crucian carp (Carassius carassius) on the growth of submerged macrophytes: Implications for shallow lake restoration. Ecological Engineering, 2016, 95: 567-573. DOI: 10.1016/j. ecoleng. 2016.06.118.

[25] Zhang XF, Liu ZW, Jeppesen E et al. Effects of benthic-feeding common carp and filter-feeding silver carp on benthic-pelagic coupling: Implications for shallow lake management. Ecological Engineering, 2016, 88: 256-264. DOI: 10.1016/j. ecoleng.2015.12.039.

[26] Qiu XC, Mei XY, Razlutskij V et al. Effects of common carp (Cyprinus carpio) on water quality in aquatic ecosystems dominated by submerged plants: a mesocosm study. Knowledge and Management of Aquatic Ecosystems, 2019, 420: 28. DOI: $10.1051 / \mathrm{kmae} / 2019017$.

[27] González-Bergonzoni I, Meerhoff M, Davidson TA et al. Meta-analysis shows a consistent and strong latitudinal pattern in fish omnivory across ecosystems. Ecosystems, 2012, 15: 492-503. DOI: 10.1007/s10021-012-9524-4.

[28] Gao J, Liu ZW, Jeppesen E. Fish community assemblages changed but biomass remained similar after lake restoration by biomanipulation in a Chinese tropical eutrophic lake. Hydrobiologia, 2014, 724: 127-140. DOI: 10.1007/s10750-0131729-9.

[29] Ye SW, Li ZJ, Lek-Ang S et al. Community structure of small fishes in a shallow macrophytic lake (Niushan Lake) along 
the middle reach of the Yangtze River, China. Aquatic Living Resources, 2006, 19 (4): 349-359. DOI: 10.1051/ alr: 2007005 .

[30] Cai YJ, Gong ZJ, Qin BQ. Standing crop and spatial distributional pattern of Mollusca in Lake Taihu, 2006-2007. J Lake $S c i, 2009,21(5)$ : 713-719. DOI: 10.18307/2009.0516. [蔡永久, 龚志军, 秦伯强. 太湖软体动物现存量及空间分 布格局(2006-2007 年). 湖泊科学, 2009, 21(5) : 713-719.]

[31] Jin XC, Tu QY eds. The standard methods in lake eutrophication investigation. Beijing: China Environmental Science Press , 1990. [ 金相灿 , 屠清瑛. 湖泊富营养化调查规范. 北京: 中国环境科学出版社, 1990. ]

[32] Yu Q, Wang JQ, Liu YQ et al. Effects of intensive Rhodeus sinensis culture on both nutritive salts and phytoplankton of water body. Acta Agriculture Shanghai, 2014, 30(4) : 52-56. [喻倩, 王金庆, 刘娅琴等. 高密度养殖鳑鲏鱼对水体营 养盐和浮游植物群落的影响. 上海农业学报, 2014, 30(4): 52-56.]

[33] Wang WL, Liu B, Han RM et al. Research advancements and perspectives on leaf and stem micro-interfaces in submerged macrophytes and its effect on water nitrogen cycling. Acta Ecologica Sinica, 2014, 34(22) : 6409-6416. DOI: 10.5846/ stxb201308152085. [王文林, 刘波, 韩睿明 等. 沉水植物茎叶微界面及其对水体氮循环影响研究进展. 生态学报, 34(22): 6409-6416.

[34] Zhou YW, Zhou XH, Han RM et al. Reproduction capacity of Potamogeton crispus fragments and its role in water purification and algae inhibition in eutrophic lakes. Science of the Total Environment, 2017, 580(15) : 1421-1428. DOI: 10. 1016/j.scitotenv.2016.12.108.

[35] He H, Jin H, Jeppesen E et al. Fish-mediated plankton responses to increased temperature in subtropical aquatic mesocosm ecosystems: Implications for lake management. Water Research, 2018, 144: 304-311. DOI: 10.1016/j.watres.2018. 07.055 .

[36] Drenner RW, Gallo KL, Baca RM et al. Synergistic effects of nutrient loading and omnivorous fish on phytoplankton biomass. Canadian Journal of Fisheries and Aquatic Sciences, 1998, 55(9) : 2087-2096. DOI: 10.1139/cjfas-55-9-2087.

[37] Zhang XF, Mei XY, Gulati RD. Effects of omnivorous tilapia on water turbidity and primary production dynamics in shallow lakes: implications for ecosystem management. Reviews in Fish Biology \& Fisheries, 2017, 27 : 245-254. DOI: 10. 1007/s11160-016-9458-6.

[38] Usui S, Kanou K, Sano M. Food habits of fishes in a freshwater reed belt in Lake Kitaura, eastern Japan, in summer. Fisheries Science, 2018, 84: 469-476. DOI: 10.1007/s12562-018-1189-2.

[39] He H, Han YQ, Li QS et al. Crucian carp (Carassius carassius) strongly affect C/N/P stoichiometry of suspended particulate matter in shallow warm water eutrophic lakes. Water, 2019, 11: 524. DOI: 10.3390/w11030524.

[40] Gu J, He H, Jin H et al. Synergistic negative effects of small-sized benthivorous fish and nitrogen loading on the growth of submerged macrophytes-Relevance for shallow lake restoration. Science of the Total Environment, 2018, 610-611: 15721580. DOI: $10.1016 /$ j. scitotenv.2017.06.119.

[41] Hilt S, Van de Weyer K, Köhler A et al. Submerged macrophyte responses to reduced phosphorus concentrations in two peri-urban lakes. Restoration Ecology, 2010, 18(S2) : 452-461. DOI: 10.1111/j.1526-100x.2009.00577.x.

[42] Cao Y, Zhang N, Sun JY et al. Responses of periphyton on non-plant substrates to different macrophytes under various nitrogen concentrations: A mesocosm study. Aquatic Botany, 2019, 154: 53-59. DOI: 10.1016/j.aquabot.2019.01.003.

[43] Dong B, Lu QP, Wang GX et al. The impact of periphyton attached on Potamogeton crispus on nitrogen and phosphorus loads in water. J Lake Sci, 2013 , 25 (3) : 359-365. DOI: 10.18307/2013.0308. [董涁, 陆全平, 王国祥等. 埧草 (Potamogeton crispus) 附着物对水体氮, 磷负荷的响应. 湖泊科学, 2013, 25(3) : 359-365.]

[44] Roberts E, Kroker J, Körner S et al. The role of periphyton during the re-colonization of a shallow lake with submerged macrophytes. Hydrobiologia, 2003, 506-509: 525-530. DOI: 10.1023/b:hydr.0000008560.73832.1c.

[45] Song YZ, Huang J, Qin BQ. Effect of epiphyte on the rapid light curves of two submerged macrophytes in Lake Taihu. $J$ Lake Sci, 2010, 22(6) : 935-940. DOI: 10.18307/2010.0618. [ 宋玉芝, 黄瑾, 秦伯强. 附着生物对太湖常见的两种 沉水植物快速光曲线的影响. 湖泊科学, 2010, 22(6): 935-940.]

[46] Jones JI, Sayer CD. Does the fish-invertebrate-periphyton cascade precipitate plant loss in shallow lakes? Ecology, 2003, 84( 8 ) : 2155-2167. DOI: $10.2307 / 3450039$.

[47] Wen MZ, Li KY, Wang CH. Effects of nutrient level on growth of Vallisneria natans in water. Research of Environmental Sciences, 2001, 21(1) : 74-77. DOI: 10.13198/j.res.2008.01.80.wenmzh.011. [ 文明章, 李宽意, 王传海. 水体的营养 水平对苦草(Vallisneria natans)生长的影响. 环境科学研究, 2008, 21(1) : 74-77.] 